\title{
Elliptic Equations, Manifolds with Non-smooth Boundaries, and Boundary Value Problems
}

\author{
Vladimir B. Vasilyev
}

To ISAAC

\begin{abstract}
We discuss basic principles for constructing the theory of boundary value problems on manifolds with non-smooth boundaries. It includes studying local situations related to model pseudo-differential equations in canonical domains. The technique consists of Fourier transform, multi-dimensional Riemann boundary value problem, wave factorization, and multi-variable complex analysis.
\end{abstract}

Keywords Elliptic symbol - Multi-dimensional Riemann boundary value problem $\bullet$ Pseudo-differential equation • Singularities $•$ Wave factorization

Mathematics Subject Classification (2010) Primary 35S15; Secondary 42B30

\section{Introduction}

One considers a general elliptic pseudo differential equation

$$
(A u)(x)=f(x), \quad x \in M,
$$

in Sobolev-Slobodetskii spaces $H^{s}(M)$, where $M$ is a smooth manifold with nonsmooth boundary, i.e. its boundary has some singularities like a cone, a wedge, etc., and the unknown function $u$ is defined on $M$.

If $A(x, \xi),(x, \xi) \in \mathbb{R}^{m} \times \mathbb{R}^{m}$, is a symbol (in local coordinates of the co-tangent bundle $T^{*} M$ ) of a pseudo-differential operator $A$, then to obtain a Fredholm property for the operator $A$ we need to describe invertibility conditions for some classes of its local representatives.

V.B. Vasilyev $(\bowtie)$

Belgorod National Research University, Studencheskaya 14/1, Belgorod 308007, Russia

e-mail: vbv57@inbox.ru 
Such operators are defined by a well-known formula if $M$ is a compact smooth manifold because one can use the "freezing coefficients principle" or, in other words, "local principle." For a manifold with a smooth boundary we need a new local formula for defining the operator $A$ : more precisely near inner points of $M$ we use an usual formula, but near boundary points we need another formula

$$
u(x) \longmapsto \int_{\mathbb{R}_{+}^{m}} \int_{\mathbb{R}^{m}} A(x, \xi) u(y) e^{i(x-y) \cdot \xi} d \xi d y .
$$

For invertibility of such an operator with symbol $A(\cdot, \xi)$ not depending on a spatial variable $x$ one can apply the theory of the classical Riemann boundary value problem for upper and lower complex half-planes with a parameter $\xi^{\prime}=$ $\left(\xi_{1}, \ldots, \xi_{m-1}\right)$. This step was systematically studied in the book [2]. But if the boundary $\partial M$ has at least one conical point, this approach is not effective.

A conical point at the boundary is such a point for which its neighborhood is diffeomorphic to the cone $C_{+}^{a}=\left\{x \in \mathbb{R}^{m}: x_{m}>a\left|x^{\prime}\right|, x^{\prime}=\left(x_{1}, \ldots, x_{m-1}\right), a>\right.$ $0\}$, hence the local definition for pseudo-differential operator near the conical point is the following

$$
u(x) \longmapsto \int_{C_{+}^{a}} \int_{\mathbb{R}^{m}} A(x, \xi) u(y) e^{i(x-y) \cdot \xi} d \xi d y
$$

To study an invertibility property for the operator (3) the author has introduced the concept of wave factorization for an elliptic symbol near a singular boundary point $[5,7,9]$ and using this property has described Fredholm properties for Eq. (1).

Other approaches to the theory of boundary value problems one can find in papers of V.G. Mazya, B.A. Plamenevskii, B.-W. Schulze, R.B. Melrose, M. Taylor, V. Nistor, and many others. I cannot enumerate all authors but in author's book [6] very large survey of these approaches with names and papers is given.

\section{Studying Model Operators}

To describe Fredholm properties for a general pseudo-differential operator on the manifold $M$ one needs to study local situations separately. These correspond to model operators in canonical domains. 


\subsection{Simple and Complicated Singularities}

\subsubsection{Simple Singularities}

A simple standard singularity in $m$-dimensional space is the cone $C_{+}^{a}$.

Example 1 A conical singularity can be stratified, i.e. for example the cone $C_{+}^{a} \times$ $C_{+}^{b} \subset \mathbb{R}^{n+m}$, where $C_{+}^{a} \subset \mathbb{R}^{n}, C_{+}^{b} \subset \mathbb{R}^{m}$, is a stratified cone

Example 2 A quadrant on the plane $\mathbb{R}^{2}$ is represented as a direct product of two half-axes.

Example 3 Octant in the space $\mathbb{R}^{3}$ is a cone of 3 -wedged angle type which can be represented as a direct product of a quadrant (i.e., two-dimensional cone) and a half-axis (one-dimensional cone).

Example 4 A wedge of codimension $k$ in $m$-dimensional space is the set $\{x \in$ $\mathbb{R}^{m}: x=\left(x^{\prime}, x^{\prime \prime}, x_{m}\right), x^{\prime} \in \mathbb{R}^{m-k}, x^{\prime}=\left(x_{1}, \ldots, x_{m-k}\right), x_{m}>a\left|x^{\prime \prime}\right|, x^{\prime \prime}=$ $\left.\left(x_{m-k+1}, \ldots, x_{m-1}\right), a>0\right\}$.

Example 5 A multi-wedged angle in $m$-dimensional space is the set $P_{m}=\{x \in$ $\left.\mathbb{R}^{m}: x_{m}>\sum_{k=1}^{m-1} a_{k}\left|x_{k}\right|, a_{k}>0\right\}$.

\subsubsection{Complicated Singularities}

Such singularities arise if a singularity's type cannot be described by the standard cone $C_{+}^{a}$.

Example 6 A variant of the thin cone $T_{m-k}=\left\{x \in \mathbb{R}^{m}: x_{m}>a\left|x^{\prime \prime}\right|, x^{\prime \prime}=\right.$ $\left.\left(x_{1}, \ldots, x_{m-k}\right), x_{m-k+1}=\cdots=x_{m-1}=0\right\}$.

Example 7 A union of $m$-dimensional cones with a common origin.

Example 8 A union of cones with distinct dimensions with a common origin.

\subsection{Local Index and Local Solvability}

Here we consider Eq. (1) for a model operator with the elliptic symbol $A(\xi)$ in a canonical $m$-dimensional domain $D$ (Examples $1-5)$. For this case we deal with a convex cone which does not contain a whole straight line. For Example 4 we have $D=C_{+}^{a} \times \mathbb{R}^{m-k}, C_{+}^{a} \subset \mathbb{R}^{k}$, and the variable $x^{\prime \prime} \in \mathbb{R}^{m-k}$ will be a parameter. Thus a principal case is that the set $D$ is a convex sharp cone in $m$-dimensional space $\mathbb{R}^{m}$. If so one needs to describe invertibility conditions for the model operator $A$ for this canonical domain. For this purpose the author has introduced a special variant 
of a multi-dimensional Riemann boundary value problem which is distinct from all known ones. This problem can be solved by using a wave factorization concept, moreover one can obtain an integral representation for the solution of the model Eq. (1). For a model equation we use "local" constructions of Sobolev-Slobodetskii spaces in $\mathbb{R}^{m}$.

\subsubsection{Spaces}

By definition the space $H^{s}(D)$ consists of distributions from the space $H^{s}\left(\mathbb{R}^{m}\right)$ [2] for which their supports belong to $\bar{D}$. A norm in the space $H^{s}(D)$ is induced by the norm of the space $H^{s}\left(\mathbb{R}^{m}\right)$. The right-hand side $f$ is chosen from the space $H_{0}^{s-\alpha}(D)$ which consists of distributions from $S^{\prime}(D)$ admitting a continuation into the whole space $H^{s-\alpha}\left(\mathbb{R}^{m}\right)$. A norm in the space $H_{0}^{s-\alpha}(D)$ is defined by the formula

$$
\|f\|_{s-\alpha}^{+}=\inf \|l f\|_{s-\alpha},
$$

where infimum is taken over all continuations $l$. Here we use notations like ones in the Eskin's book [2].

\subsubsection{Wave Factorization}

Let us denote $\stackrel{*}{D}$ a conjugate cone $[1,15]$

$$
\stackrel{*}{D}=\left\{x \in \mathbb{R}^{m}: x \cdot y>0, y \in D\right\},
$$

where $x \cdot y$ denotes an inner product.

Example 9 If $D=C_{+}^{a}$, then $\stackrel{*}{D}=\left\{x \in \mathbb{R}^{m}: a x_{m}>\left|x^{\prime}\right|\right\}$.

Definition 2.1 A radial tube domain $T(D)$ over the cone $D$ is called a subset of $m$-dimensional complex space $\mathbb{C}^{m}$ of the type $\mathbb{R}^{m}+i D$.

Definition 2.2 The symbol $A(\xi)$ is called an elliptic symbol of order $\alpha \in \mathbb{R}$ if $\exists c_{1}, c_{2}>0$ such that

$$
c_{1} \leq\left|A(\xi)(1+|\xi|)^{-\alpha}\right| \leq c_{2}, \quad \forall \xi \in \mathbb{R}^{m} .
$$

Definition 2.3 Wave factorization with respect to the cone $D$ for the elliptic symbol $A(\xi)$ is called a representation in the form

$$
A(\xi)=A_{\neq}(\xi) A_{=}(\xi)
$$


where the factors $A_{\neq}(\xi), A_{=}(\xi)$ must satisfy the following conditions:

1) $A_{\neq}(\xi), A_{=}(\xi)$ are defined for all admissible values $\xi \in \mathbb{R}^{m}$, without possibility, the points $\xi \in \partial(\stackrel{*}{D} \bigcup(-\stackrel{*}{D}))$;

2) $A_{\neq}(\xi), A_{=}(\xi)$ admit an analytical continuation into radial tube domains $T(\stackrel{*}{D})$, $T(-\stackrel{*}{D})$ respectively with estimates

$$
\begin{aligned}
& \left|A_{\neq}^{ \pm 1}(\xi+i \tau)\right| \leq c_{1}(1+|\xi|+|\tau|)^{ \pm x}, \\
& \left|A_{=}^{ \pm 1}(\xi-i \tau)\right| \leq c_{2}(1+|\xi|+|\tau|)^{ \pm(\alpha-\mathfrak{x})}, \forall \tau \in C_{+}^{*} .
\end{aligned}
$$

The number $æ \in \mathbb{R}$ is called index of wave factorization.

\subsubsection{Multi-Dimensional Riemann Problem}

Taking into account that we will use the Fourier transform let us introduce the following notations. We use notation $\tilde{u}$ for the Fourier transform of function $u$, and the notation $\widetilde{H}$ for Fourier image of the Hilbert space $H$.

For small s, $|s|<1 / 2$, we denote by $A\left(\mathbb{R}^{m}\right)$ a subspace in the space $\widetilde{H}^{s}\left(\mathbb{R}^{m}\right)$ of functions $u(x)$ which admit an analytical continuation into radial tube domain $T(\stackrel{*}{D})$ over conjugate cone $\stackrel{*}{D}$, the subspace $B\left(\mathbb{R}^{m}\right)$ is a direct complement of the subspace $A\left(\mathbb{R}^{m}\right)$ in the space $\widetilde{H}^{s}\left(\mathbb{R}^{m}\right)$, so that $\widetilde{H}^{s}\left(\mathbb{R}^{m}\right)=A\left(\mathbb{R}^{m}\right) \oplus B\left(\mathbb{R}^{m}\right)$.

The mentioned multi-variable Riemann problem is formulated as follows. One seeks two functions $\Phi^{+}(x) \in A\left(\mathbb{R}^{m}\right), \Phi^{-}(x) \in B\left(\mathbb{R}^{m}\right)$ which satisfy the linear relation

$$
\Phi^{+}(x)=W(x) \Phi^{-}(x)+w(x) .
$$

almost everywhere on $\mathbb{R}^{m}$, where $W(x), w(x)$ are given.

The transfer from Eq. (1) to the problem (4) is very simple. If we will apply the Fourier transform to the model Eq. (1) we obtain a certain multi-dimensional singular integral equation with the kernel $B(x)$ like a characteristic one-dimensional singular integral equation $[3,4]$. This kernel $B(z), z \in \stackrel{*}{D}$, is the Bochner kernel for the cone $D[1,15]$ and

$$
B(z)=\int_{D} e^{i y \cdot z} d y, \quad z=x+i \tau, \quad z \in T(\stackrel{*}{D}),
$$

and a corresponding integral operator is the following

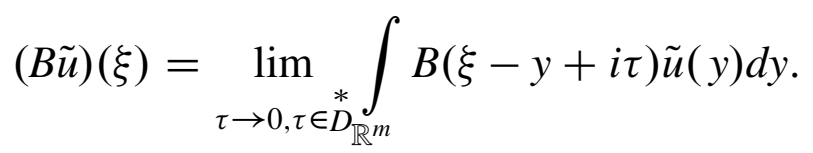


Remark 2.4 The needed variant of Paley-Wiener theorem for this situation one can find in the book [15], Chap. 5, Sect. 26. Principal point here is that representation

$$
\tilde{u}=\tilde{u}_{1}+\tilde{u}_{2}, \quad \forall \tilde{u} \in \widetilde{H}^{s}\left(\mathbb{R}^{m}\right),
$$

where $\tilde{u}_{1} \in A\left(\mathbb{R}^{m}\right), \tilde{u}_{2} \in B\left(\mathbb{R}^{m}\right)$, is unique for $|s|<1 / 2$ only (see also $[2,5]$ ).

Example 10 If $D$ is a one-dimensional cone $\mathbb{R}_{+}$, then $[1,2,15] B(z)$ is the Cauchy kernel $i(2 \pi z)^{-1}$, and the corresponding one-dimensional analogue of the singular integral operator (5) is the following

$$
\tilde{u}(\xi) \longmapsto \frac{1}{2} \tilde{u}(\xi)+\frac{1}{\pi i} v \cdot p \cdot \int_{-\infty}^{+\infty} \frac{\tilde{u}(\eta) d \eta}{\xi-\eta}
$$

This follows from Plemelj-Sokhotskii formulas [3, 4].

\subsubsection{Solvability and Boundary Conditions}

The operator $B$ and wave factorization give a possibility to describe solvability of the model Eq. (1).

Proposition 2.5 If the elliptic symbol $A(\xi)$ admits wave factorization with respect to the cone $D$ with index $æ$, then

1) for $|æ-s|<1 / 2$ there exists a unique solution $u \in H^{s}(D)$ of the model Eq. (1) for arbitrary right-hand side $f \in H_{0}^{s-\alpha}(D)$, and we have

$$
\tilde{u}(\xi)=A_{\neq}^{-1}(\xi)\left(B\left(A_{=}^{-1} \widetilde{l f}\right)\right)(\xi),
$$

where $A_{=}^{-1} \widetilde{l f}$ means the function $A_{=}^{-1}(\xi) \widetilde{l f}(\xi)$, lf is an arbitrary continuation of $f \in H_{0}^{s-\alpha}(D)$ on the whole $H^{s-\alpha}\left(\mathbb{R}^{m}\right)$;

2) for $\mathfrak{x}-s=n+\delta, n \in \mathbb{N},|\delta|<1 / 2$, there are a lot of solutions depending on $n$ arbitrary functions $c_{k} \in H^{s_{k}}\left(\mathbb{R}^{m-1}\right), s_{k}=s-\mathfrak{x}+k-1 / 2, k=1, \ldots, n$;

3) for $æ-s=-n+\delta, n \in \mathbb{N},|\delta|<1 / 2$, then a solution from $H^{s}(D)$ exists iff certain $n$ additional integral conditions on right-hand side $f \in H_{0}^{s-\alpha}(D)$ hold.

Remark 2.6 Two-dimensional variant of the proposition was proved by the author many years ago [5]. Some multi-dimensional constructions are described in [10$12]$.

Some Comments to the Proposition 2.5. Indeed functions $c_{k}$ appear after wave factorization and change of variables reducing the cone into a half-space. A certain special operator similar to a pseudo-differential one takes part in this construction. All details are given in [10-12]. 
For the case 2 of the Proposition 2.5 one needs some additional conditions to extract a unique solution. These may be boundary conditions as usual or conditions of another type. Some preliminary considerations are given in $[10,12]$.

\section{Partition of Unity and Transfer to Manifolds}

These ideas lead to many interesting deductions. To define correctly a pseudo differential operator on a manifold with non-smooth boundary one needs to choose a partition of unity and to consider boundary neighborhoods in dependence on the type of singular point. Since pseudo differential operators are operators of a local type, the Fredholm property will be conserved. It means the following. If we use a change of variables diffeomorphic transforming singular neighborhood onto certain cone we locally obtain an operator of the type (3) plus some compact operator. Since the index of an operator is stable under compact perturbations we obtain operators with same indices.

\section{Conclusion}

There are a lot of singularities types in a manifold with a non-smooth boundary. For example, the author's papers $[8,14]$ are related to thin singularities, and the paper [13] concerns to the union of cones. The author hopes that the developed methods will be useful for the general theory of boundary value problems.

Acknowledgements The author is very grateful to unknown referee for useful comments and remarks.

\section{References}

1. S. Bochner, W.T. Martin, Several Complex Variables (Princeton University Press, Princeton, 1948)

2. G. Eskin, Boundary Value Problems for Elliptic Pseudodifferential Equations (American Mathematical Society, Providence, RI, 1981)

3. F.D. Gakhov, Boundary Value Problems (Dover, New York, 1981)

4. N.I. Muskhelishvili, Singular Integral Equations (North Holland, Amsterdam, 1976)

5. V.B. Vasilyev, Wave Factorization of Elliptic Symbols: Theory and Applications (Kluwer Academic Publishers, Boston, 2000)

6. V.B. Vasilyev, Multipliers of Fourier Integrals, Pseudo-differential Equations, the Wave Factorization, Boundary Value Problems, 2nd edn. (Editorial URSS, Moscow, 2010) (in Russian)

7. V.B. Vasilyev, Elliptic equations and boundary value problems in non-smooth domains. Oper. Theory Adv. Appl. 213, 105-121 (2011) 
8. V.B. Vasilyev, Asymptotical analysis of singularities for pseudo differential equations in canonical non-smooth domains, in Integral Methods in Science and Engineering. Computational and Analytic Aspects (Birkhäuser, Boston, 2011), pp. 379-390

9. V.B. Vasilyev, Pseudo differential equations on manifolds with non-smooth boundaries, in Differential and Difference Equations and Applications. Springer Proceedings in Mathematics and Statistics, vol. 47 (Springer, New York, 2013), pp. 625-637

10. V.B. Vasilyev, On the Dirichlet and Neumann problems in multi-dimensional cone. Math. Bohem. 139, 333-340 (2014)

11. V.B. Vasilyev, On certain elliptic problems for pseudo differential equations in a polyhedral cone. Adv. Dyn. Syst. Appl. 9, 227-237 (2014)

12. V.B. Vasilyev, New constructions in the theory of elliptic boundary value problems, in Integral Methods in Science and Engineering. Theoretical and Computational Advances (Birkhäuser, Basel, 2015), pp. 629-641

13. V.B. Vasilyev, Pseudodifferential equations in cones with conjugation points on the boundary. Differ. Equ. 51, 1113-1125 (2015)

14. V.B. Vasilyev, On asymptotical expansions for certain singular integrals: 2-dimensional case (2015), pp. 1-9, arXiv:1512.08237

15. V.S. Vladimirov, Methods of the Theory of Functions of Many Complex Variables (Dover, Mineola, 2007) 